\title{
Smoking is a risk factor for endogenous peritonitis in patients undergoing peritoneal dialysis
}

Kohsuke Terada ${ }^{1}$, Yuichiro Sumi ${ }^{1}$, Sae Aratani ${ }^{1}$, Akio Hirama ${ }^{1}$, Tetsuya Kashiwagi ${ }^{1}$, and Yukinao Sakai $^{1}$

${ }^{1}$ Department of Nephrology, Graduate School of Medicine, Nippon Medical School, Tokyo 113-8603, Japan

Corresponding author: Yukinao Sakai

Department of Nephrology, Graduate School of Medicine, Nippon Medical School 1-1-5 Sendagi, Bunkyo-ku, Tokyo 113-8603, Japan

Tel: +81-3-3822-2131 ext. 6496

Fax: $+81-3-3822-4865$

Email: y-sakai@nms.ac.jp

K Terada: k-terada@nms.ac.jp, Y Sumi: smith0821@nms.ac.jp, S Aratani: saearatani@nms.ac.jp, A Hirama: ahirama@nms.ac.jp, T Kashiewagi: tk@nms.ac.jp

Short title: Smoking is a risk factor for peritonitis 


\begin{abstract}
Background: Peritonitis is one of the most common complications in patients who are undergoing peritoneal dialysis (PD). However, it is difficult to predict or prevent the onset of endogenous peritonitis. In this study, we investigated the risk of developing endogenous peritonitis in patients receiving PD.
\end{abstract}

Methods: We included all of the patients who underwent PD at our hospital from April 2015 to March 2020. There were 22 cases of peritonitis, including 18 cases of endogenous peritonitis without evidence of exit-site infection or technical failure. We considered older age, female sex, obesity, diabetes mellitus, diverticulosis, and constipation as important risk factors for endogenous peritonitis. We included these as confounding factors with a current or previous history of smoking in univariate logistic regression models.

Results: In this study, previous or current history of smoking $(p=0.0065)$ was the most significant risk factor for endogenous peritonitis in univariate logistic regression model. In addition, smoking was also the most significant independent risk factor for endogenous peritonitis $(\mathrm{p}=0.0034)$ in our multivariate logistic regression models. It was not primary objective that diabetes mellitus was also significant in univariate and multivariate logistic regression analysis.

Conclusions: Smoking is the significant independent risk factor for endogenous peritonitis in patients undergoing PD. The discontinuation of smoking may lower the risk of endogenous peritonitis in this patient group.

Keywords: endogenous peritonitis, smoking, diabetes mellitus, peritoneal dialysis 


\section{Introduction}

Peritonitis is one of the most common complications in patients receiving peritoneal dialysis (PD). PD-related peritonitis is associated with total mortality and transfer from PD to hemodialysis ${ }^{1-4}$. Moreover, peritonitis is associated with a higher risk of cardiovascular and infection-related mortality in patients undergoing $\mathrm{PD}^{2}$. Therefore, the prediction and prevention of peritonitis are important in these patients. The International Society for Peritoneal Dialysis published the "ISPD Peritonitis Recommendations: 2016 Update on Prevention and Treatment" for patients undergoing PD. Many risk factors for PD-related peritonitis were highlighted in the guidelines and in other reports ${ }^{1,3-8}$. Specifically, exit-site infection (ESI) is a risk factor for peritonitis and reducing the occurrence of ESI is important for preventing PD-related peritonitis ${ }^{3,9}$. However, endogenous peritonitis may present in patients receiving PD without evidence of ESI or technical failure ${ }^{10}$. Consequently, the prevention and prediction of endogenous peritonitis is important. Research has shown that smoking is a risk factor for PD-related peritonitis ${ }^{1,3-8}$. However, it is unclear if smoking is a risk factor for endogenous peritonitis. In our hospital, we investigated all cases of endogenous peritonitis in current and former smokers undergoing PD and evaluated the risk of them developing endogenous peritonitis.

\section{Methods}

We investigated all patients who underwent PD at our hospital from April 2015 to March 2020. We treated 22 patients with peritonitis, including 18 cases of endogenous peritonitis without evidence of ESI or technical failure. Some factors, such as those shown in Table 1, have been previously reported as risk factors for peritonitis , $^{1,8,11-16}$. 
We considered older age, female sex, obesity, diabetes mellitus, diverticulosis, and constipation as important risk factors for endogenous peritonitis in patients undergoing PD. We added these six factors as confounding factors with a current or previous history of smoking in univariate logistic regression models.

\section{Statistical analyses}

Statistical analyses were performed using Prism ${ }^{\circledR}$ software version 8 (GraphPad Software, La Jolla, CA, USA). Multiple linear and logistic regression analyses were performed using JMP ${ }^{\circledR}$ software version 12 (SAS Institute, Cary, NC, USA). Laboratory values are presented as mean \pm standard deviation and $p$ values $<0.05$ were considered statistically significant.

\section{Results}

This study included 17 men and 5 women, with a mean age of $64.6 \pm 12.3$ years. The most frequent primary condition, seen in 17 patients, was type 2 diabetes mellitus (77.3\%). Table 2 shows the baseline characteristics of all patients.

\section{Risk factors for endogenous peritonitis}

We used univariate logistic regression models for smoking, older age, female sex, obesity, diabetes mellitus, diverticulosis, and constipation. We defined older age as those patients over 65 years old and obesity as a body mass index $>25$. We defined patients who received laxatives at onset of PD as having constipation. 
In this study, previous or current history of smoking $(\mathrm{p}=0.0065)$ was the most significant risk factor for endogenous peritonitis in the univariate logistic regression models. It was not the primary aim that diabetes mellitus $(\mathrm{p}=0.0106)$ was also a statistically significant risk factor for endogenous peritonitis in the univariate logistic regression models.

For the multivariate logistic regression models for risk of endogenous peritonitis, we selected smoking and diabetes mellitus as the significant factors in the multivariate logistic regression models. Smoking was the most significant independent risk factor for endogenous peritonitis $(\mathrm{p}=0.0034)$ in our multivariate logistic regression model. It was not the primary objective that diabetes mellitus $(p=0.0055)$ was also a significant independent risk factor for endogenous peritonitis in this study.

The results are presented in Table 3.

\section{Discussion}

\section{PD-related peritonitis and risk factors}

Peritonitis is one of the most common complications in patients undergoing PD and leads to discontinuation of PD and potential fatality ${ }^{1-4}$. Peritonitis is associated with morbidity and mortality caused by cardiovascular events and infections independently ${ }^{2}$. Therefore, the correct management of peritonitis in patients receiving PD is crucial. Our hospital manages patients undergoing PD according to the International Society for Peritoneal Dialysis' 2016 guidelines, which describe the risk factors for developing PDrelated peritonitis ${ }^{3}$. Although many risk factors for PD-related peritonitis have been 
reported, currently, only topical exit-site antimicrobial prophylaxis and nasal eradication of Staphylococcus aureus have provided sufficient evidence in terms of the reduction in rates of peritonitis in such patients ${ }^{4}$. Moreover, the risk factors for developing endogenous peritonitis in this patient group remain unclear. It is important to predict and prevent endogenous peritonitis as it may present without evidence of ESI or technical failure.

\section{PD-related peritonitis and smoking}

Smoking is a modifiable cardiovascular risk factor and is significantly associated with mortality in patients receiving PD ${ }^{17-19,20}$. In contrast, smoking is not associated with cardiovascular events in patients receiving PD or hemodialysis, despite a reduction in all-cause mortality ${ }^{18}$. The guidelines published by the International Society for Peritoneal Dialysis described smoking as a risk factor for peritonitis, and additional studies have confirmed this ${ }^{1.3-8}$. However, to our knowledge, no report has clearly described the mechanisms that explain the association of smoking and PD-related peritonitis. In one study, the major cause of peritonitis in current and former smokers was gram-positive cocci ${ }^{8}$. The authors state that this might be due to the management of hygiene and/or care with sterile, no-touch disconnection techniques for the patients.

One study has reported that silent diverticulosis detected using computed tomography is not a risk factor for enteric diverticulosis-related peritonitis ${ }^{21}$. However, another study has shown that diverticulosis may be a risk factor for peritonitis ${ }^{12}$. Whether diverticulosis increases the risk of peritonitis in patients receiving PD remains controversial, however, smoking is a significant risk factor for colonic diverticulosis ${ }^{22}$. 
In addition, smoking is associated with complicated diverticulitis and a worse prognosis, and smoking cessation decreases the rate of diverticulitis recurrence ${ }^{23,24}$. Moreover, smoking is an independent prognostic factor for colon cancer ${ }^{25}$, is associated with a failure of gas exchange in the lungs, and is linked to systemic and intestinal ischemia ${ }^{26}$. In addition, smoking causes angiogenesis and failure in the gastrointestinal tract epithelial barrier ${ }^{26}$. Smoking affects both the innate and adaptive immunity, worsening the pathogenic immune response or defensive immunity ${ }^{27}$. Some studies have shown that smoking affects the intestinal microbiome and may lead to the development of intestinal and systemic diseases, such as inflammatory bowel disease ${ }^{28}$. Moreover, smoking affects the skin and delays cutaneous wound healing caused by dysfunction of the innate immune response in the $\operatorname{skin}^{29}$.

In our hospital, there were 18 cases of endogenous peritonitis without evidence of ESI or technical failure. Smoking was a significant risk factor for endogenous peritonitis in both the univariate and multivariate logistic regression models in this study. As previously stated, the harmful effects of smoking are associated with compromised peritoneal health that may increase the risk of endogenous peritonitis. Moreover, we should be mindful of the harmful effect of smoking on the skin around the exit site. Smoking may also cause ESI and ESI-rerated peritonitis in patients undergoing PD, although this association was unclear in our study. Further research in this patient group is warranted to confirm this finding.

\section{PD-related peritonitis and diabetes mellitus}

Previous guidelines and studies have shown that diabetes mellitus is a risk factor for 
peritonitis in patients undergoing $\mathrm{PD}^{1,3-6,11,30}$. It was not primary objective that diabetes mellitus was the second most significant risk factor for endogenous peritonitis after smoking and constipation in our univariate and multivariate logistic regression models. Patients with diabetes mellitus who receive PD are at risk of developing peritonitis, potentially owing to dysfunction of the immune system and technical problems caused by visual impairment ${ }^{31}$. Diabetes mellitus is associated with carcinogenesis $^{32}$ and, moreover, patients may have cutaneous microbiome dysbiosis that could be a risk factor for cutaneous infection ${ }^{33}$. Although the mechanism of association of diabetes mellitus and endogenous peritonitis is unclear, diabetes mellitus was a significant risk factor for endogenous peritonitis in this study. It is possible that diabetes mellitus is associated not only with peritonitis caused by ESI and technical failure, but also with endogenous peritonitis. Further research is necessary to confirm these findings.

In this study, smoking was the most significant independent risk factor for endogenous peritonitis the univariate and multivariate logistic regression models. It was not primary objective that diabetes mellitus was also a significant independent risk factor for endogenous peritonitis. Current smoking in patients undergoing PD is the treatable and modifiable factor, and the management of the factor may prevent the development of endogenous peritonitis. The prediction and prevention of endogenous peritonitis needs to be considered in addition to ESI in smokers undergoing PD.

\section{Limitations}

This study has certain limitations. Firstly, the study was conducted at a single center and 
the number of participants was insufficient to allow robust statistical analysis. Secondly, the diagnosis of endogenous peritonitis was made clinically in certain patients, without investigatory confirmation. These limitations may have introduced various biases in our results.

\section{Conclusions}

In conclusion, smoking is the significant independent risk factor for endogenous peritonitis in patients receiving PD. The management of the discontinuation of smoking may lower the risk of such patients developing endogenous peritonitis.

\section{Data availability}

All data generated or analyzed during this study are available from the corresponding author upon reasonable request.

\section{Statement of ethics}

The study protocol was approved by the Ethical Committee of Nippon Medical School Hospital (B-2020-122) and designed in accordance with the Declaration of Helsinki. The study was registered to the University Hospital Medical Information Network (UMIN No.000041046).

\section{Consent}

All participants signed written informed consent forms, which included information about the research. Confidentiality of information and patient anonymity were adhered 
to in this study.

\section{Conflicts of interest}

The authors have no conflicts of interest to declare.

\section{Funding}

None

\section{Authors' contributions}

KT drafted the first manuscript. KT, YSu, SA, AH, TK, and YSa managed the patients. YSa coordinated the data analysis and helped with writing the manuscript. All authors participated in discussions and read and approved the final manuscript.

\section{Acknowledgments}

The authors are grateful to all of the participants and medical staff of Nippon Medical School Hospital Blood Purification Center. 


\section{References}

[1] Mehrotra R, Devuyst O, Davies SJ, Johnson DW. The Current State of Peritoneal Dialysis. J Am Soc Nephrol. 2016 Nov;27(11):3238-3252. Available from: https://www.ncbi.nlm.nih.gov/pubmed/27339663.

[2] Ye H, Zhou Q, Fan L, et al. The impact of peritoneal dialysis-related peritonitis on mortality in peritoneal dialysis patients. BMC Nephrol. 2017 Jun 5;18(1):186. Available from: https://www.ncbi.nlm.nih.gov/pubmed/28583107.

[3] Li PK, Szeto CC, Piraino B, et al. ISPD Peritonitis Recommendations: 2016 Update on Prevention and Treatment. Perit Dial Int. 2016 Sep 10;36(5):481-508. Available from: https://www.ncbi.nlm.nih.gov/pubmed/27282851.

[4] Cho Y, Johnson DW. Peritoneal dialysis-related peritonitis: towards improving evidence, practices, and outcomes. Am J Kidney Dis. 2014 Aug;64(2):278-289. Available from: https://www.ncbi.nlm.nih.gov/pubmed/24751170.

[5] McDonald SP, Collins JF, Rumpsfeld M, Johnson DW. Obesity is a risk factor for peritonitis in the Australian and New Zealand peritoneal dialysis patient populations. Perit Dial Int. 2004 Jul-Aug;24(4):340-346. Available from: https://www.ncbi.nlm.nih.gov/pubmed/15335147.

[6] See EJ, Johnson DW, Hawley CM, et al. Early Peritonitis and Its Outcome in Incident Peritoneal Dialysis Patients. Perit Dial Int. 2017 Sep 28. Available from: https://www.ncbi.nlm.nih.gov/pubmed/28970365.

[7] Karagulle IV, Kaynar K, Ulusoy S, Ozkan G, Cansiz M. Risk factors for peritonitis related to peritoneal dialysis. Bratisl Lek Listy. 2013;114(9):531-533. Available from: https://www.ncbi.nlm.nih.gov/pubmed/24020711.

[8] Kotsanas D, Polkinghorne KR, Korman TM, Atkins RC, Brown F. Risk factors 
for peritoneal dialysis-related peritonitis: can we reduce the incidence and improve patient selection? Nephrology (Carlton). 2007 Jun;12(3):239-245. Available from: https://www.ncbi.nlm.nih.gov/pubmed/17498118.

[9] van Diepen AT, Tomlinson GA, Jassal SV. The association between exit site infection and subsequent peritonitis among peritoneal dialysis patients. Clin $\mathrm{J}$ Am Soc Nephrol. 2012 Aug;7(8):1266-1271. Available from: https://www.ncbi.nlm.nih.gov/pubmed/22745277.

[10] Suh H, Wadhwa NK, Cabralda T, Sorrento J. Endogenous peritonitis and related outcome in peritoneal dialysis patients. Adv Perit Dial. 1996;12:192-195. Available from: https://www.ncbi.nlm.nih.gov/pubmed/8865900.

[11] Bolton L. Preventing Peritoneal Dialysis Infections. Wounds. 2019 Jun;31(6):163-165. Available from: https://www.ncbi.nlm.nih.gov/pubmed/31215869.

[12] Yip T, Tse KC, Lam MF, et al. Colonic diverticulosis as a risk factor for peritonitis in Chinese peritoneal dialysis patients. Perit Dial Int. 2010 MarApr;30(2):187-191. Available from:

https://www.ncbi.nlm.nih.gov/pubmed/20124196.

[13] Kosmadakis G, Albaret J, Da Costa Correia E, Somda F, Aguilera D. Constipation in Peritoneal Dialysis Patients. Perit Dial Int. 2019 SepOct;39(5):399-404. Available from: https://www.ncbi.nlm.nih.gov/pubmed/30852519.

[14] Su CY, Pei J, Lu XH, Tang W, Wang T. Gastrointestinal symptoms predict peritonitis rates in CAPD patients. Clin Nephrol. 2012 Apr;77(4):267-274. Available from: https://www.ncbi.nlm.nih.gov/pubmed/22445469. 
[15] Singharetnam W, Holley JL. Acute treatment of constipation may lead to transmural migration of bacteria resulting in gram-negative, polymicrobial, or fungal peritonitis. Perit Dial Int. 1996 Jul-Aug;16(4):423-425. Available from: https://www.ncbi.nlm.nih.gov/pubmed/8863339.

[16] Afsar B, Elsurer R, Bilgic A, Sezer S, Ozdemir F. Regular lactulose use is associated with lower peritonitis rates: an observational study. Perit Dial Int. 2010 Mar-Apr;30(2):243-246. Available from: https://www.ncbi.nlm.nih.gov/pubmed/20200371.

[17] Zaza G, Rugiu C, Trubian A, Granata S, Poli A, Lupo A. How has peritoneal dialysis changed over the last 30 years: experience of the Verona dialysis center. BMC Nephrol. 2015 Apr 14;16:53. Available from: https://www.ncbi.nlm.nih.gov/pubmed/25885318.

[18] Liebman SE, Lamontagne SP, Huang LS, Messing S, Bushinsky DA. Smoking in dialysis patients: a systematic review and meta-analysis of mortality and cardiovascular morbidity. Am J Kidney Dis. 2011 Aug;58(2):257-265. Available from: https://www.ncbi.nlm.nih.gov/pubmed/21664017.

[19] Braatvedt GD, Rosie B, Bagg W, Collins J. Current and former smoking increases mortality in patients on peritoneal dialysis. N Z Med J. 2006 May 19;119(1234):U1977. Available from: https://www.ncbi.nlm.nih.gov/pubmed/16718288.

[20] Foley RN, Herzog CA, Collins AJ. Smoking and cardiovascular outcomes in dialysis patients: the United States Renal Data System Wave 2 study. Kidney Int. 2003 Apr;63(4):1462-1467. Available from: https://www.ncbi.nlm.nih.gov/pubmed/12631362. 
[21] Toda S, Ito Y, Mizuno M, et al. Asymptomatic diverticulosis identified by computed tomography is not a risk factor for enteric peritonitis. Nephrol Dial Transplant. 2012 Jun;27(6):2511-2516. Available from: https://www.ncbi.nlm.nih.gov/pubmed/22189207.

[22] Wijarnpreecha K, Boonpheng B, Thongprayoon C, Jaruvongvanich V, Ungprasert P. Smoking and risk of colonic diverticulosis: A meta-analysis. J Postgrad Med. 2018 Jan-Mar;64(1):35-39. Available from: https://www.ncbi.nlm.nih.gov/pubmed/29067919.

[23] Wilkins T, Embry K, George R. Diagnosis and management of acute diverticulitis. Am Fam Physician. 2013 May 1;87(9):612-620. Available from: https://www.ncbi.nlm.nih.gov/pubmed/23668524.

[24] Turunen P, Wikstrom H, Carpelan-Holmstrom M, Kairaluoma P, Kruuna O, Scheinin T. Smoking increases the incidence of complicated diverticular disease of the sigmoid colon. Scand J Surg. 2010;99(1):14-17. Available from: https://www.ncbi.nlm.nih.gov/pubmed/20501352.

[25] Sharp L, McDevitt J, Brown C, Comber H. Smoking at diagnosis significantly decreases 5-year cancer-specific survival in a population-based cohort of 18166 colon cancer patients. Aliment Pharmacol Ther. 2017 Mar;45(6):788-800. Available from: https://www.ncbi.nlm.nih.gov/pubmed/28176335.

[26] Fricker M, Goggins BJ, Mateer S, et al. Chronic cigarette smoke exposure induces systemic hypoxia that drives intestinal dysfunction. JCI Insight. 2018 Feb 8;3(3). Available from: https://www.ncbi.nlm.nih.gov/pubmed/29415878.

[27] Qiu F, Liang CL, Liu H, et al. Impacts of cigarette smoking on immune responsiveness: Up and down or upside down? Oncotarget. 2017 Jan 3;8(1):268- 
284. Available from: https://www.ncbi.nlm.nih.gov/pubmed/27902485.

[28] Savin Z, Kivity S, Yonath H, Yehuda S. Smoking and the intestinal microbiome. Arch Microbiol. 2018 Jul;200(5):677-684. Available from:

https://www.ncbi.nlm.nih.gov/pubmed/29626219.

[29] Maarouf M, Maarouf CL, Yosipovitch G, Shi VY. The impact of stress on epidermal barrier function: an evidence-based review. Br J Dermatol. 2019 Dec;181(6):1129-1137. Available from: https://www.ncbi.nlm.nih.gov/pubmed/30614527.

[30] Tsai CC, Lee JJ, Liu TP, et al. Effects of age and diabetes mellitus on clinical outcomes in patients with peritoneal dialysis-related peritonitis. Surg Infect (Larchmt). 2013 Dec;14(6):540-546. Available from: https://www.ncbi.nlm.nih.gov/pubmed/24116738.

[31] Benabed A, Bechade C, Ficheux M, Verger C, Lobbedez T. Effect of assistance on peritonitis risk in diabetic patients treated by peritoneal dialysis: report from the French Language Peritoneal Dialysis Registry. Nephrol Dial Transplant. 2016 Apr;31(4):656-662. Available from:

https://www.ncbi.nlm.nih.gov/pubmed/26932691.

[32] Wojciechowska J, Krajewski W, Bolanowski M, Krecicki T, Zatonski T. Diabetes and Cancer: a Review of Current Knowledge. Exp Clin Endocrinol Diabetes. 2016 May;124(5):263-275. Available from: https://www.ncbi.nlm.nih.gov/pubmed/27219686.

[33] Thimmappaiah Jagadeesh A, Prakash PY, Karthik Rao N, Ramya V. Culture characterization of the skin microbiome in Type 2 diabetes mellitus: A focus on the role of innate immunity. Diabetes Res Clin Pract. 2017 Dec;134:1-7. 
Available from: https://www.ncbi.nlm.nih.gov/pubmed/28951341.

\section{Legends}

Table 1: Risk factors for PD-related peritonitis

PD, peritoneal dialysis.

Table 2: Patients' baseline characteristics and parameters

BMI, body mass index; RASi, renin angiotensin system inhibiter; Vit D, vitamin D; PD, peritoneal dialysis; CVA, cerebrovascular accident; eGFR, estimated glomerular filtration rate; Alb, serum albumin; CRP, C-reactive protein.

Table 3: Logistic regression analysis for endogenous peritonitis 


\section{Risk factors for peritoneal dialysis-related peritonitis}

\section{Characteristics of patients}

Older age

Female sex

Lower socioeconomic status

Smoking

Obesity

Pets

Living further from PD unit

\section{Comorbidities}

Diabetes mellitus

Depression

\section{Clinical status}

Hypoalbuminemia

Hypokalemia

Absence of vitamin D supplementation

\section{Others}

Nasal Staphylococcus aureus carrier status

Previous exit-site infection

Invasive medical interventions (e.g. colonoscopy)

Patient training 


\begin{tabular}{|c|c|}
\hline total, $\mathrm{n}$ & 22 \\
\hline Female, n (\%) & $5(22.7)$ \\
\hline Age (years) & $66.6 \pm 12.3$ \\
\hline BMI $\left(\mathrm{kg} / \mathrm{m}^{2}\right)$ & $23.7 \pm 4.0$ \\
\hline Smoking, n (\%) & $13(59.1)$ \\
\hline Diatbetes, n (\%) & $17(77.3)$ \\
\hline RASi, n (\%) & $12(54.5)$ \\
\hline Diuretic-use $(\%)$ & $11(50.0)$ \\
\hline Vit D supplementation at onset of PD, n (\%) & $7(31.8)$ \\
\hline Malignant tumor, n (\%) & $9(40.9)$ \\
\hline CVA, n $(\%)$ & $4(18.2)$ \\
\hline Hepatitis, n (\%) & $4(18.2)$ \\
\hline History of abdominal operation, $\mathrm{n}(\%)$ & $9(40.9)$ \\
\hline Diverticulosis, n (\%) & $11(50.0)$ \\
\hline Pancreatic cyst, n (\%) & $6(27.3)$ \\
\hline Gallstone, n (\%) & $8(36.4)$ \\
\hline Laxative at onset of PD, $\mathrm{n}(\%)$ & $4(18.2)$ \\
\hline Laxative at onset of petironitis, $\mathrm{n}(\%)$ & $12(54.5)$ \\
\hline Phosphate binder at onset of PD, n (\%) & $5(22.7)$ \\
\hline Phosphate binder at onset of petironitis, $\mathrm{n}(\%)$ & $13(59.1)$ \\
\hline eGFR at start of PD (mL/ min/1.73m^2) & $7.57 \pm 5.48$ \\
\hline Serum K (mEq/L) & $4.64 \pm 0.61$ \\
\hline Serum Alb (g/dL) & $3.18 \pm 0.70$ \\
\hline $\mathrm{CRP}(\mathrm{mg} / \mathrm{dL})$ & $0.47 \pm 1.22$ \\
\hline $\mathrm{D} / \mathrm{P}$ ratio & $0.71 \pm 0.15$ \\
\hline D/D0 ratio & $0.37 \pm 0.10$ \\
\hline
\end{tabular}


Logistic regression analysis for endogenous peritonitis

\begin{tabular}{|c|c|c|c|c|}
\hline \multirow{2}{*}{ Variables } & \multicolumn{2}{|c|}{ Univariate analysis } & \multicolumn{2}{|l|}{ Multivariate analysis } \\
\hline & Odds ratio $(95 \% \mathrm{CI})$ & $p$ value & Odds ratio $(95 \% \mathrm{CI})$ & $p$ value \\
\hline Older age & $0.5238095(0.0232836-5.0904939)$ & 0.5931 & & \\
\hline Female & $0.2(0.0168925-2.1865373)$ & 0.1777 & & \\
\hline Obesity & $1.4999998(0.1514566-33.961144)$ & 0.7419 & & \\
\hline Diabetes & $23.999996(2.0503527-658.36579)$ & 0.0106 & $75847820(1.83 e+126-4.88 e+140)$ & 0.0055 \\
\hline Smoking & $92131825(2.7235235$ - ND) & 0.0065 & $1.135 e+14(5.9276547-\mathrm{ND})$ & 0.0034 \\
\hline Diverticulosis & $3.75(0.3924605-84.23298)$ & 0.2599 & & \\
\hline Constipation & $0.6(0.0508106-14.409328)$ & 0.7051 & & \\
\hline
\end{tabular}

